\section{Efecto de la dieta y otros factores ambientales sobre el riesgo de morir de cáncer de próstata}

Ciertos factores ambientales, como la dieta y el hábito de fumar, están asociados con 60 a 70\% de la incidencia de cáncer y con la mortalidad por esta causa. En el caso del cáncer de próstata, la dieta desempeña un papel especialmente importante, y las tasas de mortalidad por cáncer de próstata varían considerablemente entre países que poseen diferentes hábitos alimentarios. Por ejemplo, la mortalidad por cáncer de próstata en países del norte de Europa es cinco veces mayor que en Hong Kong, Irán, Japón o Turquía. Una mejor comprensión de la relación entre la dieta y el cáncer de próstata puede ayudar a prevenir esta enfermedad.

Aunque existe consenso acerca del papel que desempeñan algunos factores dietéticos en el cáncer de próstata, todavía hay discrepancias de opinión en cuanto a otros factores y a su importancia relativa. El objetivo de esta investigación fue determinar la importancia relativa de la dieta y algunos otros elementos ambientales en el riesgo de morir de cáncer de próstata, así como identificar los factores que pueden ayudar a reducir este riesgo.

Se aplicó un enfoque ecológico multinacional mediante un análisis de regresión multifactorial en el que se tomaron en cuenta las tasas de mortalidad por cáncer de próstata, factores dietéticos y las cantidades anuales de radiaciones solares ultravioleta $B$ (UV-B) en cada lugar. Se obtuvieron de la Organización Mundial de la Salud las tasas de mortalidad por cáncer de próstata de 32 países con poblaciones constituidas predominantemente por personas de raza blanca, desglosadas por países y ajustadas por edad según la distribución de edades de la población mundial. Los datos relacionados con la dieta se obtuvieron de la Organización de las Naciones Unidas para la Alimentación y la Agricultura. Las dosis anuales de radiación UV-B se obtuvieron de las estaciones terrenas europeas. Se realizaron análisis de regresión lineal independientes para los 32 países estudiados y los 20 países europeos.

El análisis se restringió a un grupo de países con poblaciones predominantemente blancas a fin de evitar la influencia de factores genéticos y del estilo de vida, que varían mucho entre personas de diferentes grupos étnicos y raciales. Otros criterios de inclusión fueron una población mayor de 3,5 millones de habitantes, una esperanza de vida superior a 60 años y la disponibilidad de información dietética correspondiente al período comprendido entre 1979 y 1981. La amplia variabilidad dietética entre los diferentes países facilitó la aplicación de un enfoque ecológico en este análisis.

Los factores estudiados fueron el consumo de alcohol, cerveza, vino, cereales, huevos, productos energéticos de origen animal y vegetal; el consumo energético total; la ingestión de grasas de origen animal y vegetal y de frutas, legumbres, carne, leche descremada, cebolla, proteína de origen animal y vegetal, edulcorantes, tomate y otras hortalizas. También se analizó la dosis anual de radiación UV-B recibida por los habitantes de cada país. Adicionalmente, en el estudio se tomó en cuenta el consumo de algunas combinaciones de alimentos, tales como los "productos protectores de origen vegetal" (todos menos el alcohol, las grasas y los edulcorantes).

Se emplearon los datos de la radiación UV-B anual en 11 países situados entre los $28^{\circ}$ y $69^{\circ}$ de latitud norte para generar un gráfico con la dosis de radiación recibida anualmente en cada latitud, ajustada mediante una curva cuadrática. La sensibilidad espectral del sensor de radiación UV-B empleado era cercana al del espectro de acción eritémica.

El análisis de la relación entre la dieta y la mortalidad por cáncer de próstata en los países europeos reveló la presencia de una correlación inversa entre el consumo de cebolla y el cáncer de próstata. Otros vegetales también mostraron un efecto protector. En cambio, se observó una débil relación directa entre el consumo de grasa, leche descremada y otros productos de origen animal y la mortalidad por cáncer de próstata. La dosis anual de radiación UV-B y el consumo de tomate presentaron una débil correlación inversa con dicha mortalidad.

El análisis del conjunto de países estudiados (tanto los europeos como los de otros continentes) demostró que el consumo de productos de origen animal presenta la mayor correlación con la mortalidad por cáncer de próstata, mientras que la ingestión de cebolla y de productos vegetales mostraron una correlación inversa. Además, el consumo de leche descremada y de alcohol mostró una asociación directa significativa con la mortalidad por cáncer de próstata. El consumo de cereales mostró una correlación indirecta significativa similar a la encontrada en el caso de otros productos protectores de origen vegetal. La exposición a radiación UV-B siguió mostrando carácter protector. 
Estos resultados confirman los de otras investigaciones en el sentido de que algunos compuestos de la cebolla parecen poseer un efecto protector que también se ha demostrado en conexión con otros tipos de cáncer, tales como los mielomas y el cáncer de páncreas. Sin embargo, en el estudio de todos los países no se confirmó el efecto protector que se suele atribuir a las hortalizas que contienen licopeno en abundancia, como el tomate.

Algunos factores, como el consumo de edulcorantes y tomates y la dosis de radiación UV-B recibida anualmente, tuvieron efectos favorables o adversos en relación con la mortalidad por cáncer de próstata cuando se consideraron de forma independiente, pero la correlación dejó de observarse en el análisis multifactorial. A pesar de que en otros estudios de casos y testigos y de cohortes estos factores se han asociado con el cáncer de próstata, es posible que esto se deba a su vinculación con otros factores que se asocian más directamente con este tipo de cáncer, confundiéndose así su efecto verdadero en investigaciones con un enfoque ecológico.

En este estudio, el consumo de alcohol estuvo débilmente correlacionado con la mortalidad por cáncer de próstata en el conjunto de países estudiados, pero no en los países europeos. Esto puede deberse a que el primer grupo abarca algunos países del Mediano Oriente donde no se consume alcohol. Por lo tanto, a pesar de este resultado contradictorio se puede afirmar que el consumo de alcohol constituye un factor de riesgo de cáncer de próstata. Por otra parte, la asociación encontrada entre el consumo de productos de origen animal, leche descremada y alcohol, y la mortalidad por cáncer de próstata, así como el efecto protector de las hortalizas y de la radiación UV-B, se compaginan con la asociación directa observada entre el factor de crecimiento insulinoide I (FCI-I) y el cáncer de próstata.

Estos resultados deben tomarse en cuenta para el diseño de estudios dirigidos a evaluar la relación entre la dieta y otros factores ambientales con el cáncer de próstata. Sin embargo, debido a que el tiempo necesario para observar el efecto de un cambio dietético en la mortalidad por cáncer de próstata es de 15 a 20 años, la confirmación de estos resultados mediante estudios basados en la modificación de la dieta puede demorar. (Grant WB. A multicountry ecologic study of risk and risk reduction factors for prostate cancer mortality. Eur Urol. 2004;45: 271-279.)

\section{Asociación entre el tabaquismo y el glaucoma primario de ángulo abierto}

A pesar de que el glaucoma primario de ángulo abierto (GPAA) es una de las principales causas de ceguera y de mala visión, aún no se conocen los mecanismos causales de esta enfermedad. Estudios realizados demuestran que la edad avanzada, los antecedentes familiares de glaucoma y el pertenecer a la raza negra son factores de riesgo de GPAA; sin embargo, aún se debate si el tabaquismo también lo es.

En este estudio se examinó la asociación entre el hábito de fumar cigarrillos y la GPAA mediante un metaanálisis de los estudios publicados en revistas científicas con arbitraje por pares. Se identificaron todos los estudios sobre el tema publicados entre el 1 de enero de 1966 y el 31 de diciembre de 2002. La pesquisa se hizo en MEDLINE, empleando como término de búsqueda la combinación de glaucoma con cigarette, smoking y tobacco en el título, las palabras clave y el texto. Los resultados se evaluaron para excluir los artículos ajenos al tema y para evitar posibles sesgos.

Se tomaron en cuenta los estudios de casos y testigos, de cohortes y transversales que evaluaran la asociación entre el GPAA y el consumo de cigarrillos. Se excluyeron los artículos que: 1) no estuvieran escritos en inglés; 2) no hubieran considerado el hábito de fumar como factor de riesgo de GPAA; 3) no tuvieran suficientes datos para determinar algún estimador del riesgo relativo o los intervalos de confianza; o 4) no especificaran que la enfermedad estudiada era GPAA.

Prácticamente todos los estudios clasificaron a las personas estudiadas en "fumadores actuales", "ex fumadores" y "no fumadores" (personas que nunca fumaron). De cada artículo se tomaron los datos del estudio (fecha de realización, primer autor, año de publicación y país de la población estudiada), el diseño del estudio y el número de personas estudiadas, así como las razones de posibilidades (odds ratios, RP) y sus respectivos intervalos de confianza de 95\% (IC95\%).

Se ajustaron dos modelos metaanalíticos para comparar las posibilidades de contraer GPAA de los "fumadores actuales" frente a los "no fumadores", y las de los "ex fumadores" frente a los "no fumadores".

En total se identificaron 108 artículos en MEDLINE, pero solo 10 de ellos estudiaban la asociación entre el consumo de cigarrillos y la GPAA, y únicamente siete cumplían todos los criterios de inclusión.

La prueba de la $Q$ de Cochran aplicada a los dos modelos metaanalíticos no dio resultados estadísticamente significativos, lo cual indica que los resultados de los estudios eran homogéneos. Sin embargo, el reducido número de investigaciones tomadas en cuenta y los pequeños tamaños muestrales limitaron el alcance de este análisis de heterogeneidad.

El resumen de los efectos fijos del primer modelo metaanalítico reveló una posibilidad $37 \%$ mayor de 
contraer GPAA entre los "fumadores actuales" $(\mathrm{RP}=1,37$; IC95\%: 1,00 a 1,87; $P=0,050)$ que entre personas que nunca fumaron. En cambio, el resumen de los efectos fijos del segundo modelo indicó que los "ex fumadores" no corrían un riesgo mayor de contraer GPAA que los "no fumadores" (RP = 1,03; IC95\%: 0,77 a 1,38; $P=0,85$ ).

Este es el primer metaanálisis en el que se evalúa si el consumo de cigarrillos constituye un factor de riesgo de GPAA. Según estos resultados, los fumadores de cigarrillos están en mayor riesgo de padecer esta enfermedad que las personas que nunca han fumado. A diferencia de otros factores de riesgo conocidos, el hábito de fumar es un factor modificable, por lo que en las campañas contra el tabaquismo debe hacerse hincapié en el posible riesgo de contraer GPAA. (Bonovas S, Filioussi K, Tsantes A, Peponis V. Epidemiological association between cigarette smoking and primary open-angle glaucoma: a meta-analysis. Public Health. 2004;118: 256-261.)

\section{Características de la infección invasora por Haemophilus influenzae tipo b en Paraguay}

Hasta 1997, Haemophilus influenzae tipo b (Hib) era la causa de 25 a 50\% de los casos anuales de meningitis bacteriana aguda (MBA) en América Latina, con una tasa de mortalidad de $40 \%$. Esta situación epidemiológica ha venido cambiando en los últimos años gracias a la incorporación de la vacuna contra Hib en los programas de vacunación pública de la mayoría de los países latinoamericanos. Sin embargo, en los países de menos recursos, donde el uso de esta vacuna no se ha generalizado, la infección causada por esta bacteria - en primer lugar la meningitis bacteriana- aún constituye la causa principal de morbilidad y mortalidad en lactantes y niños. Además, esta bacteria causa otros trastornos y enfermedades, como septicemia, artritis, epiglotitis, celulitis y neumonía, y ha adquirido resistencia a varios antibióticos, especialmente en los países en desarrollo.

El objetivo de este estudio fue documentar las características clínicas y epidemiológicas de la enfermedad invasora causada por Hib en niños de Paraguay.

Se realizó un análisis retrospectivo de todos los pacientes menores de 15 años de edad con un diagnóstico de infección invasora por Hib, según criterios clínicos y de laboratorio, que fueron atendidos en el Instituto de Medicina Tropical (IMT) de Asunción, Paraguay, entre enero de 1991 y septiembre de 1995.

Se anotaron los datos demográficos, clínicos y microbiológicos de los casos que cumplieron con los criterios de inclusión. Los pacientes se presentaron con un diagnóstico clínico de meningitis, epiglotitis, neumonía, pericarditis, bacteriemia oculta, celulitis, artritis séptica u osteomielitis. La infección se confirmó por métodos bacteriológicos, mediante el aislamiento de Hib en la sangre, en el líquido cefalorraquídeo (LCR) o en otro líquido o tejido estéril (derrame pleural, sinovial o pericárdico, muestras de tejido óseo, de piel, o de tejidos blandos) o mediante la detección de antígenos contra Hib en el LCR (solamente en casos de meningitis).

Los pacientes se clasificaron de acuerdo con el tipo de infección inicial, meníngea o extrameníngea. En el grupo de pacientes con meningitis, se consideraron complicaciones los trastornos cardíacos o cerebrovasculares repentinos, los abscesos cerebrales y el derrame subdural. Las secuelas en el momento del alta hospitalaria fueron hidrocefalia, afectación de los nervios craneales, pérdida auditiva y retraso psicomotor o mental (definido como un retraso del desarrollo de 6 meses o más). La deficiencia auditiva se definió como ligera si el umbral auditivo era de 30 a $40 \mathrm{~dB}$, moderada si estaba entre 50 y $70 \mathrm{~dB}$ y grave cuando era mayor de $70 \mathrm{~dB}$.

En el período estudiado se atendió en el IMT a 102 pacientes pediátricos (58 niños y 44 niñas) con infección invasora por Hib. Se atendieron de 19 a 29 casos anuales, con excepción del año 1994, en el que solo se atendió a 10 pacientes. Durante los 5 años estudiados, las tasas anuales de infección invasora, meningitis e infección extrameníngea en niños menores de 5 años de edad del área de la Gran Asunción fueron de 21,6, 17,7 y 3,9 por 100 000, respectivamente. En todos los años, menos 1994, se observó poca variación en el número de casos registrados anualmente.

La edad media de los pacientes fue de 15,3 \pm 12 meses (entre 1 mes y 14 años). De ellos, 50\% eran menores de 1 año de edad y $80 \%$ menores de 2 años. Solo 3 casos (3\%) se encontraban en el grupo de edad de 5 a 14 años. En general, 36\% de los pacientes (37/102) tenían desnutrición y 32\% (12/37), desnutrición grave. Ningún paciente estaba vacunado contra Hib. De los 102 pacientes, 83 (81\%) tenían MBA y 19 (19\%), infección extrameníngea. Ambos grupos se analizaron por separado.

La infección meníngea se diagnosticó en los 83 pacientes mediante el aislamiento microbiológico o la detección de antígenos de Hib en el LCR. En 71 de estos casos (85\%), el sistema nervioso central fue el sitio de la infección. Doce niños $(14 \%)$ tuvieron otras infecciones: neumonía (10 casos), osteomielitis con artritis adyacente (un caso) y celulitis (un caso). La edad media de los pacientes con meningitis fue de 15,3 \pm 14,3 meses; 46 (55\%) de ellos eran niños y $37(45 \%)$ eran niñas. El grupo de edad más afectado por la MBA fue el de menores de 1 año (45 pacien- 
tes, $54 \%$ ). Veinte pacientes (24\%) tenían menos de 6 meses de edad.

De los casos con meningitis, 26 pacientes (31\%) presentaron desnutrición, que fue grave en dos de los casos. El tiempo transcurrido entre la aparición de los síntomas y el diagnóstico de meningitis fue de 1 a 7 días (mediana $=4,9 \pm 3,9$ días). La mediana del tiempo transcurrido entre la aparición de los síntomas y el ingreso al hospital fue de 5,7 $\pm 4,3$ días en los niños de 1 a 11 meses de edad y de 5,6 3,7 días en los niños de 12 a 23 meses; en cambio, este período fue de 3,9 $\pm 2,9$ días en los niños de 24 a 60 meses de edad. No obstante, estas diferencias no fueron estadísticamente significativas. Antes de las 24 horas recibieron tratamiento con antibióticos 28 pacientes $(34 \%)$. Se encontró resistencia a la ampicilina en 30\% de las cepas aisladas de Hib; $20 \%$ de las cepas mostraron resistencia al cloranfenicol y $10 \%$ a ambos antibióticos.

Las manifestaciones clínicas más frecuentes fueron la fiebre $(89 \%)$, los vómitos (58\%), los trastornos cardíacos y cerebrovasculares repentinos (54\%) y las afecciones sensoriales de origen central (54\%). Estas manifestaciones fueron similares en todos los grupos de edad.

La mortalidad general por meningitis fue de $13 \%$ y disminuyó a medida que aumentaba la edad: de $22 \%(10 / 45)$ en pacientes menores de 12 meses de edad a $2,6 \%(1 / 38)$ en niños mayores $(P<0,02)$. No se encontraron diferencias significativas en el tiempo transcurrido entre la aparición de los síntomas y el inicio del tratamiento entre los niños que murieron $(6,5 \pm 4,6$ días) y los niños que sobrevivieron $(4,6 \pm 1,9 ; P>0,1)$.

La concentración de glucosa en el LCF en el momento de la consulta al hospital fue el mejor factor pronóstico de mortalidad. De los pacientes fallecidos, $91 \%$ presentaban concentraciones de glucosa en el LCF menores de $10 \mathrm{mg} / \mathrm{dL}$ en el momento del ingreso al hospital, mientras que solo $61 \%$ de los pacientes que sobrevivieron (44/72) presentaban concentraciones de glucosa de $10 \mathrm{mg} / \mathrm{dL}$ o menos $(P<0,05)$.

Se observaron secuelas graves en 39\% de los pacientes sobrevivientes (28/72). Los sobrevivientes menores de 24 meses de edad presentaron una o varias de las siguientes secuelas: pérdida auditiva, hidrocefalia moderada o grave y retraso mental moderado o grave. Las secuelas a largo plazo fueron más frecuentes en los niños menores de 12 meses (51\%) seguidos de los de 24 a 60 meses de edad (42\%).

La infección extrameníngea por Hib se observó en $19 \%$ de los pacientes (19/102), de los cuales $79 \%$ (15/19) tenían neumonía. De estos casos, 87\% (13/15) tenían derrame pleural. Se logró aislar Hib de la sangre de 9 pacientes; del líquido pleural de 13 , y de ambos en 6 casos. La distribución por eda- des fue similar a la observada en los casos de meningitis (73\% eran niños menores de 24 meses y $27 \%$ tenían entre 24 y 60 meses de edad). En $20 \%$ de los casos de neumonía por Hib se encontraron trastornos del sistema nervioso central, lo cual indica la utilidad de realizar una punción lumbar en todos los casos de infección invasora por Hib.

Los resultados del presente trabajo indican que, a diferencia de lo encontrado en otros lugares, la infección por Hib en Paraguay afecta principalmente a los niños durante el primer año de la vida (50\% de los casos) y especialmente durante los primeros 6 meses (24\% de todos los casos notificados). Esto significa que el esquema de vacunación recomendado por la Academia Estadounidense de Pediatría (a los 2, 4 y 6 meses de edad, con una dosis de refuerzo a los 12-15 meses) podría ser inapropiado para el Paraguay. Se deben evaluar otras opciones, como la inmunización materna, o establecer esquemas de vacunación a edades más tempranas, por ejemplo, a los 4, 8-12 y 16 semanas, con una dosis de refuerzo a los 12-15 meses. (Basualdo W, Arbo A. Invasive Haemophilus influenzae type b infections in children in Paraguay. Arch Med Res. 2004;35:126-133.)

\section{Validación cultural de instrumentos para el diagnóstico de la demencia en países en desarrollo}

Independientemente de los criterios que se observen para diagnosticar la demencia, el diagnóstico se debe basar en tres parámetros: el deterioro de al menos dos dominios de la función cognoscitiva, incluida la memoria; la afectación del funcionamiento social u ocupacional del paciente; y la ausencia de otro diagnóstico que explique los síntomas, como el de depresión.

Según el método estándar para el diagnóstico de la demencia en dos etapas, los instrumentos de tamizaje cognoscitivo pueden identificar a la mayoría de las personas que no padecen de demencia. Sin embargo, en los países en desarrollo, la baja escolaridad, el analfabetismo y la falta de familiaridad con la aritmética en amplios sectores de la población pueden llevar al diagnóstico erróneo de demencia en personas que tienen limitaciones cognoscitivas. Para superar esa dificultad es necesario adaptar los instrumentos de tamizaje a poblaciones de bajo nivel cultural y educacional, de manera que no sea un requisito que la persona evaluada sepa leer, escribir o realizar operaciones aritméticas.

Este trabajo describe los resultados obtenidos durante un estudio piloto multicéntrico multinacional en el que se evaluaron los resultados de la aplicación de instrumentos para el diagnóstico de la demencia, adaptados para su uso con personas de bajo 
nivel cultural y educacional. Estos instrumentos se basaron en la prueba para determinar el estado mental geriátrico (GMS) que utiliza el programa de computación AGECAT, y en el instrumento para el tamizaje de demencia en la comunidad (CSI-D).

En este estudio, el Grupo de Investigación de la Demencia 10/66 entrevistó a personas de 60 años o más en 25 centros - universitarios en su mayor parte- ubicados en 15 países. En cada centro se trató de reclutar a 30 participantes para cada uno de los siguientes cuatro grupos: pacientes con demencia ligera a moderada (criterios de gravedad de demencia según el Manual diagnóstico y estadístico de trastornos mentales [cuarta edición, DSM-IV], y de demencia ligera a moderada según la escala de clasificación de demencia clínica); con depresión (18 puntos o más según la escala de clasificación de la depresión de Montgomery Asberg), pero sin signos de demencia; con alta escolaridad y sin signos de demencia; y con baja escolaridad y sin signos de demencia. Las personas de los últimos dos grupos se reclutaron en la comunidad o en organizaciones para adultos mayores y se siguieron los criterios de escolaridad de los propios centros.

En total participaron 2885 personas: 760 de la India, 367 de China y el Sudeste Asiático, 76 de Nigeria y 1682 de América Latina y el Caribe (Argentina, Brasil, Chile, Cuba, Guatemala, México, Panamá, Perú, República Dominicana, Uruguay y Venezuela). Del total de personas encuestadas, 729 tenían un diagnóstico de demencia. El grupo con un diagnóstico de depresión se compuso de 702 personas; el grupo que tenía alta escolaridad, de 694 personas; y el grupo con baja escolaridad, de 760 personas. La proporción de personas sin educación o con una escolaridad mínima fue de $91 \%$ en la India, 89\% en la China y el Sudeste Asiático, y 80\% en América Latina y el Caribe. En esos mismos lugares, la proporción de personas que habían completado la educación secundaria fue de $81 \%$, 99\% y $80 \%$, respectivamente. Todas las personas que participaron en la investigación vivían en la comunidad y tenían un informante que podía acompañarlos a las entrevistas.

Un entrevistador que desconocía el diagnóstico de demencia de los entrevistados aplicó cuatro pruebas ajustadas y validadas culturalmente: el CSI-D; la prueba para evaluar el GMS mediante AGECAT; la prueba de fluidez verbal para nombrar animales de distintas clases; y una prueba de aprendizaje de una lista de 10 palabras, modificada por el Consorcio para el Establecimiento de un Registro de la Enfermedad de Alzheimer (CERAD). Para garantizar que los entrevistadores no conocieran el diagnóstico de los participantes, estos fueron reclutados y diagnosticados por médicos locales que no participaron en la evaluación subsiguiente.
El CSI-D mostró una buena capacidad discriminatoria. Sin embargo, no permitió distinguir claramente entre la demencia y la depresión ni —aunque en menor grado- entre la demencia y la baja escolaridad. Esta prueba tuvo mayor sensibilidad y especificidad en la India que en China o en América Latina. En la mayor parte de los centros, independientemente de la región, la puntuación funcional discriminatoria (DFSCORE) del CSI-D, combinada con la puntuación de la función cognoscitiva (COGSCORE) y la puntuación según la información brindada por el informante que acompañaba a cada paciente (RELSCORE), logró una mejor discriminación que el COGSCORE solamente y redujo el número de resultados positivos falsos en los grupos de personas con depresión y baja escolaridad.

En general, la prueba GMS/AGECAT mostró una precisión aceptable para el diagnóstico de la demencia en dos etapas (jerárquico). Esta prueba logró identificar a 505 personas $(75 \%)$ con demencia y a 512 personas $(78 \%$ ) con depresión. Sin embargo, a 91 personas $(13 \%)$ del grupo con baja escolaridad se les diagnósticó demencia por error, al igual que a 99 personas $(15 \%)$ del grupo con depresión.

La prueba de fluidez verbal para nombrar animales de distintas clases no identificó adecuadamente el estado de demencia. Se observó un marcado efecto de la escolaridad independientemente de la región, y los resultados obtenidos en personas con depresión no fueron satisfactorios.

La prueba de aprendizaje de una lista de 10 palabras permitió identificar un estado de demencia, aunque con resultados modestos. El recuerdo diferido reflejó el estado de demencia mejor que el recuerdo inmediato y se vio menos afectado por la escolaridad.

Los instrumentos para el diagnóstico de la demencia evaluados en este trabajo, los cuales se adaptaron para personas de bajo nivel cultural y educacional, permitieron mejorar la sensibilidad y especificidad del diagnóstico. La única excepción fue la prueba de fluidez verbal para nombrar animales por categorías. Empleadas en conjunto, estas pruebas permitieron llegar al diagnóstico correcto, tanto en los pacientes con demencia (94\%) como en otras personas: $85 \%$ de las personas con depresión, $97 \%$ de las que tenían alta escolaridad y $94 \%$ de las que tenían baja escolaridad. Poder distinguir entre los casos de demencia y de depresión continúa siendo difícil, ya que la prueba resultó en un diagnóstico erróneo de demencia en 15\% de las personas con depresión.

El algoritmo propuesto constituye una sólida base para el diagnóstico de la demencia mediante instrumentos adaptados al nivel cultural y educacional de las personas. Tales instrumentos tienen utilidad clínica y en investigaciones poblacionales, 
han sido traducidos a la mayoría de los idiomas de los países en desarrollo y están disponibles para otras investigaciones, especialmente en poblaciones con baja escolaridad. Asimismo, se prestan para realizar comparaciones válidas entre poblaciones de diferentes países y con distintas culturas. (Prince M, Acosta D, Chiu H, Scazufca M, Varghese M. Dementia diagnosis in developing countries: a crosscultural validation study. Lancet. 2003;361(9361): 909-17.)

\section{La inmunidad provoca el aumento de la virulencia en un modelo experimental de malaria}

Diversas investigaciones han revelado la presencia de cambios genéticos que se asocian con la evolución de la virulencia en organismos patógenos, es decir, con el aumento o la disminución de su capacidad para causar daños al organismo hospedero. Numerosos mecanismos pueden facilitar la adquisición de rasgos genéticos que modifican la virulencia de los organismos patógenos. Por ejemplo, la inmunidad del hospedero contra el patógeno - ya sea adquirida de forma natural o mediante la vacunación- puede evitar que aquel muera, lo cual le permite al patógeno vivir más tiempo y tener más oportunidad de evolucionar. De esta forma, en poblaciones de personas con un alto grado de inmunidad es factible que surjan cepas patógenas más virulentas que las halladas en poblaciones de personas sin inmunidad.

La inmunidad de la población también puede ejercer una presión selectiva dentro del propio hospedero, como resultado de una competencia entre organismos patógenos con diferentes grados de virulencia. Lamentablemente, no se conocen los mecanismos de esta selección.

En este estudio se utilizó un modelo experimental de malaria murina (causada por Plasmodium chabaudi) para determinar si la presión selectiva ejercida por la inmunidad lleva al agente patógeno a adquirir mayor virulencia. En el estudio se utilizaron dos líneas progenitoras de $P$. chabaudi (una virulenta y otra inocua) y ratones hembras C57BI/6J de 7 a 10 semanas de edad. Cinco sublíneas de cada línea progenitora del parásito se pasaron repetidamente por ratonas inmunizadas y sin inmunizar, con lo que se establecieron 20 sublíneas en total. Las pasadas se realizaron mediante la inoculación de
$0,1 \mathrm{~mL}$ de sangre diluida que contenía $5 \times 10^{5}$ parásitos obtenida de ratonas donantes que habían sido infectadas siete días antes, es decir, se obvió el paso del parásito por el mosquito vector. Después de 20 pasadas se compararon el grado de virulencia y la capacidad de transmisión de las sublíneas parasitarias que habían pasado por las ratonas inmunizadas y las de las sublíneas parasitarias que habían pasado por las ratonas sin inmunizar. Seguidamente se probó si el aumento de la virulencia se mantenía cuando el parásito era transmitido por un mosquito.

Tanto las sublíneas de parásitos sometidas a 20 pasadas sucesivas en las ratonas inmunizadas como las pasadas por las ratonas sin inmunizar mostraron una mayor virulencia que la población parasitaria progenitora. El aumento de la virulencia fue mayor en las sublíneas derivadas de las ratonas inmunizadas. Cuando después de las 20 pasadas los parásitos se transmitieron mediante mosquitos, se observó una reducción de la virulencia en todas las sublíneas, pero las sublíneas obtenidas mediante pases en ratonas inmunizadas mantuvieron una mayor virulencia que las obtenidas en las ratonas sin inmunizar.

Estos resultados demuestran que la inmunidad del huésped actúa como una poderosa fuerza selectiva dentro del hospedero y que esta presión refuerza la virulencia del parásito infectante. Sin embargo, se requieren investigaciones adicionales que expliquen el mecanismo que provoca estos cambios. Como la inmunización evita la muerte del hospedero, las variantes más virulentas del parásito se ven favorecidas por la presión selectiva interna, lo que puede provocar la diseminación de líneas más virulentas en poblaciones de hospederos inmunizados.

La presión selectiva ejercida por la inmunidad del huésped aceleró en las líneas de Plasmodium la adquisición de mayor virulencia. Este hecho, unido a la conocida capacidad de este parásito de adaptarse rápidamente a nuevas condiciones de laboratorio y al efecto de la inmunización con vacunas específicas contra diferentes variantes, confirman la necesidad de mantener un seguimiento constante de la virulencia de Plasmodium en poblaciones vacunadas contra la malaria. (Mackinnon M, Read AF. Immunity promotes virulence evolution in a malaria model. PLoS Biol. 2004;2(9):e230. Hallado en:http://www.plosbiology.org/plosonline/ ?request $=$ get - document $\&$ doi $=10.1371 /$ journal . pbio. 0020230. Acceso el 23 de junio de 2004.) 